\title{
Finasteride in Frontal Fibrosing Alopecia: Joining the Dots!
}

\author{
Arshdeep $^{a}$ Raj Kubba ${ }^{a}$ Wilma F. Bergfeld ${ }^{b}$ Chakravarthi Rangachari Srinivas ${ }^{c}$ \\ ${ }^{a}$ Delhi Dermatology Group, New Delhi, India; ${ }^{b}$ Department of Dermatology, Cleveland Clinic, Cleveland, OH, USA; \\ 'Department of Dermatology, KIMS, Bhubaneswar, Orissa, India
}

\section{Dear Editor,}

5-alpha reductase inhibitors ( $5 \mathrm{aRI}$ ) such as finasteride and dutasteride have increasingly shown the best efficacy in the stabilization and improvement of frontal fibrosing alopecia (FFA) and also reversal of atrophy $[1,2]$. A hormonal basis of the disease pathogenesis has been emphasized earlier on the basis of the prevalence of FFA in postmenopausal women with a high rate of early menopause or hysterectomy noted in some studies [1], which probably led to therapeutic trials of $5 \mathrm{aRI}$ in FFA. However, the mode of action of these antiandrogens in an autoimmune disease remains elusive. Whether it treats the co-existing androgenetic alopecia (AGA) and enhances the residual hair density, or whether it has a direct role in arresting the lichenoid disease process is yet undefined. We hereby attempt to give a plausible explanation for the same.

FFA is a variant of lichen planopilaris (LPP), affecting predominantly the marginal scalp and eyebrows. The exact pathogenesis is unknown, but it is believed that there is preferential involvement of vellus-like/intermediate hairs, unlike terminal hairs, by the lymphocytic inflammatory infiltrate and fibrosis in FFA, as shown by Tosti et al. [3]. The susceptibility of vellus and intermediate hairs to FFA is a subject of debate, as these hairs are otherwise also more numerous in FFA-affected areas. In addition, whether it is linked to follicle size or shorter anagen duration is yet to be understood. Nevertheless, finasteride, by inhibiting the conversion of testosterone to dihydro-testosterone, causes reversal of miniaturization; i.e., it converts vellus/intermediate hairs to terminal hairs as well as prolongs the anagen duration. Furthermore,

karger@karger.com

(c) 2020 S. Karger AG, Basel

www.karger.com/sad

Karger! these "terminalized" hairs then can escape the assault of the lichenoid pathology. Hence, finasteride with its antiandrogenic action, can contribute to the "follicular rescue" process in this scarring alopecia.

This hypothesis applies in certain other androgen-dependent areas as well. FFA is known to involve the occipital scalp margin also, where the role of finasteride would at first seem dubious, as the occipital region of the scalp represents an androgen-independent area. Ryu et al. [4] demonstrated that the ratio of the scalp skin dihydrotestosterone to testosterone does not decrease with finasteride in the occipital scalp. Interestingly, the lower occipital area; i.e., the occipital margin of the scalp, is actually involved in miniaturization as part of AGA, as was originally described in male-pattern hair loss by Hamilton [5] in 1951, which has somehow lost attention in recent times. Hence, finasteride, by reversal of miniaturization, acts by arresting the progression of FFA at the occipital scalp as well.

In recent years, the prevalence of FFA is being increasingly recognized in premenopausal women and men [6]. A study on serum sex hormone levels in 43 premenopausal women has reported no consistent alterations, suggesting that sex hormone levels are not directly implicated in the pathogenesis of FFA [7]. However, a potential hormonal involvement by a local mechanism; i.e., endorgan hypersensitivity to androgens, as is noted in AGA, cannot be excluded. Hence, the above hypothesis on the role of $5 \mathrm{aRI}$ in FFA holds true for the treatment in premenopausal women as well, but these drugs must be administered with adequate contraceptive measures. 
Fibrosing alopecia in pattern distribution (FAPD) is another recently described variant of LPP, wherein the frontal scalp is diffusely involved in scarring [8]. FAPD shares many features with FFA, as both represent scarring alopecia, but in a distribution of a typically female-pattern and male-pattern AGA, respectively [9]. Hence, the selective involvement of miniaturized hairs by lichenoid inflammatory infiltrate seems likely in both these entities. Zinkernagel and Trüeb [8] have reported improvement with finasteride in a patient of FAPD. Finasteride has also been tried successfully by Mardones et al. [10] in 4 women who were not stabilized using topical minoxidil and clobetasol alone. This can also be explained by a similar mechanism of action of finasteride, namely, to help rescue "predisposed" vellus/intermediate follicular units by reversing the miniaturization, as in FFA.

While the above points clearly support our hypothesis on the beneficial role of finasteride in FFA, on the other hand, there are some contradictory findings in the literature. Firstly, eyebrow improvement has been reported by Georgala et al. [11] in 6 out of 7 patients of FFA after 1 year of oral dutasteride. This cannot be explained by the above-mentioned hypothesis on finasteride, as this drug cannot transform a normal vellus-like/intermediate hair like that of an eyebrow, into a terminal follicle. Secondly, although vellus/intermediate hairs have been observed to be more commonly affected, terminal hairs are also affected at the same time. The mechanism of action of $5 a$ RIs on terminal hairs is difficult to explain. Hence, further studies need to be directed to decipher the exact pathogenesis of the disease and therapeutic role of these drugs.
By far, the above plausible hypothesis on the role of finasteride in FFA and FAPD only highlights the underlying pathophysiology of these two diseases. Unlike LPP, only the target vellus/intermediate hair follicles in FFA might express certain biological markers that induce a lymphocytic reaction leading to follicular destruction and scarring $[3,12]$. As also highlighted by Olsen [9] in 2005, further research needs to be done to unveil the potential role of androgens in the development of inflammatory reaction in the scalp. Till then, these drugs offer a good therapeutic option to halt the disease process in an indirect way.

\section{Statement of Ethics}

The authors have no ethical conflicts to disclose.

\section{Disclosure Statement}

The authors have no conflicts of interest to declare.

\section{Funding Sources}

No funding was sought for this work.

\section{Author Contributions}

The statements, opinions, and data contained in this publication are solely those of the individual authors and contributors. Arshdeep has contributed in all stages of the paper. Raj Kubba, Wilma F. Bergfeld, and C.R. Srinivas have contributed in the concept, design, revision, and final approval of the paper.

\section{References}

1 Vañó-Galván S, Molina-Ruiz AM, SerranoFalcón C, Arias-Santiago S, Rodrigues-Barata AR, Garnacho-Saucedo G, et al. Frontal fibrosing alopecia: a multicenter review of 355 patients. J Am Acad Dermatol. 2014 Apr; 70(4):670-8.

2 Donovan JC. Finasteride-mediated hair regrowth and reversal of atrophy in a patient with frontal fibrosing alopecia. JAAD Case Rep. 2015 Sep;1(6):353-5.

3 Tosti A, Piraccini BM, Iorizzo M, Misciali C. Frontal fibrosing alopecia in postmenopausal women. J Am Acad Dermatol. 2005 Jan;52(1): 55-60.

4 Ryu HK, Kim KM, Yoo EA, Sim WY, Chung BC. Evaluation of androgens in the scalp hair and plasma of patients with male-pattern baldness before and after finasteride administration. Br J Dermatol. 2006 Apr;154(4):730-4.
5 Hamilton JB. Patterned loss of hair in man; types and incidence. Ann N Y Acad Sci. 1951 Mar;53(3):708-28.

6 Starace M, Brandi N, Alessandrini A, Bruni F, Piraccini BM. Frontal fibrosing alopecia: a case series of 65 patients seen in a single Italian centre. J Eur Acad Dermatol Venereol. 2019 Feb;33(2):433-8.

7 Bernárdez C, Molina-Ruiz AM, Vañó-Galvan $S$, Urech M, Saceda-Corralo D, Moreno-Arrones OM, et al. Sex hormone status in premenopausal women with frontal fibrosing alopecia: a multicentre review of 43 patients. Clin Exp Dermatol. 2017 Dec;42(8):921-3.

8 Zinkernagel MS, Trüeb RM. Fibrosing alopecia in a pattern distribution: patterned lichen planopilaris or androgenetic alopecia with a lichenoid tissue reaction pattern? Arch Dermatol. 2000 Feb;136(2):205-11.
9 Olsen EA. Female pattern hair loss and its relationship to permanent/cicatricial alopecia: a new perspective. J Investig Dermatol Symp Proc. 2005 Dec;10(3):217-21.

10 Mardones F, Hott K, Martinez MC. Clinical study of fibrosing alopecia in a pattern distribution in a Latin American population. Int J Dermatol. 2018 Feb;57(2):e12-4.

11 Georgala S, Katoulis AC, Befon A, Danopoulou I, Georgala C. Treatment of postmenopausal frontal fibrosing alopecia with oral dutasteride. J Am Acad Dermatol. 2009 Jul; 61(1):157-8.

12 Harries MJ, Meyer K, Chaudhry I, E Kloepper J, Poblet E, Griffiths CE, et al. Lichen planopilaris is characterized by immune privilege collapse of the hair follicle's epithelial stem cell niche. J Pathol. 2013 Oct;231(2): 236-47. 